Revue internationale P.M.E.

Économie et gestion de la petite et moyenne entreprise

\title{
L'insertion de la PMI dans le Management logistique de la grande entreprise : Une application sectorielle
}

\section{Gilles Paché}

Volume 2, numéro 1, 1989

URI : https://id.erudit.org/iderudit/1007904ar

DOI : https://doi.org/10.7202/1007904ar

Aller au sommaire du numéro

Éditeur(s)

Presses de l’Université du Québec

ISSN

0776-5436 (imprimé)

1918-9699 (numérique)

Découvrir la revue

Citer cet article

Paché, G. (1989). L'insertion de la PMI dans le Management logistique de la grande entreprise : Une application sectorielle. Revue internationale P.M.E., 2(1), 7-18. https://doi.org/10.7202/1007904ar
Résumé de l'article

Consciente des limites de l'intégration verticale, la grande entreprise industrielle cherche aujourd'hui à développer des transactions marchandes avec de multiples organisations. Les chaînes de valeur reviennent ainsi de plus en plus désintégrées et ce sont des PMI qui assurent l'exploitation de certains maillons. A travers l'étude de cas concrets, l'article montre que ces manoeuvres stratégiques concourent à l'émergence de nouveaux modèles organisationnels. Le management logistique y joue un rôle essentiel pour garantir la coordination des opérations sous-traitées auprès de PMI. 


\title{
L'insertion de la PMI dans le Management logistique de la grande entreprise : Une application sectorielle
}

\author{
Gilles PACHE \\ Université de Reims Champagne-Ardenne
}

\begin{abstract}
RESUME
Consciente des limites de l'intégration verticale, la grande entreprise industrielle cherche aujourd'hui à développer des transactions marchandes avec de multiples organisations. Les chaînes de valeur reviennent ainsi de plus en plus désintégrées et ce sont des PMI qui assurent l'exploitation de certains maillons. A travers l'étude de cas concrets, l'article montre que ces manoeuvres stratégiques concourent à l'émergence de nouveaux modèles organisationnels. Le management logistique y joue un róle essentiel pour garantir la coordination des opérations sous-traitées auprès de PMI.
\end{abstract}

\begin{abstract}
Aware of the limits of vertical integration, the big industrial firm nowadays tries to increase market transactions with numerous organizations. Thus, the value chains become more and more disintegrated and so small-and-medium-sized businesses are engaged in exploiting some links. Through the study of actual cases, this article shows that these strategies converge to the emergence of new organizational patterns. Logistics management here plays an essential part in the co-ordination of sub-contracted operations by small-and-medium-sized businesses.
\end{abstract}

- Gilles Paché est Maître de Conférences à l'Université de Reims Champagne-Ardenne et chercheur au CRET (Université d'Aix-Marseille 2). Auteur de nombreux articles sur les stratégies logistiques, il a publié, en collaboration, La logistique de distribution (Chotar et Associés, Paris, 1988) et a participé à l'ouvrage collectif Information et organisation spatiale (Paradigme, Caen, 1988). Son adresse personnelle est : M. Gilles Pache, 59, Allée du Bois de l'Etrier, 77350 Le Mée sur Seine, France. Première version reçue en février 1989. 
L'insertion de la PMI dans le Management logistique de la grande entreprise :

\section{RESUMEN}

Conscientes de los límites de la integración vertical, la gran empresa industrial, hoy día, intenta desarrollar transacciones comerciales con varias organizaciones. Las cadenas de valor se vuelven asi cada vez más desintegradas y son las pequeñas y medianas empresas (las PyMES) las que aseguran la explotación de algunas mallas. A través del estudio de casos concretos, el articulo muestra que estas estrategias contribuyen a la emergencia de nuevos modelos organizacionales. En este caso, el management logístico desempeña un papel esencial para garantizar la coordinacón de las operaciones subcontratadas con las PyMES. 
Depuis quelques années, les économies occidentales font l'objet d'une double mutation. D'un côté, de grandes entreprises industrielles, souvent multinationales, renoncent aux stratégies d'intégration verticale : elles privilégient de plus en plus des relations marchandes (non hiérarchiques) entre elles et un ensemble hétérogène de sous-traitants, fournisseurs et prestataires de services. De l'autre, la petite et moyenne industrie voit son rôle reconnu dans la dynamique économique, sa «flexibilité» étant particulièrement appréciée dans un environnement qui réclame désormais d'importantes capacités d'adaptation à court, voire très court terme.

Ces deux phénomènes, étroitement corrélés, ont été partiellement décrits par des travaux antérieurs (cf. par exemple Marchesnay et alii, 1979). Cependant, le chercheur en gestion dispose à l'heure actuelle d'outils d'analyse pour les replacer dans une perspective générale. Certes, beaucoup reste à faire pour proposer un cadre conceptuel décrivant les différentes interactions stratégiques entre les grandes firmes et les PMI. Tout au moins peut-on s'engager sur quelques pistes de recherche permettant de mieux comprendre quelques-unes de ces interactions.

C'est l'objet de cet article qui souhaite mesurer l'impact du management logistique, c'est-à-dire, en première approximation, la maîtrise de la circulation physique des marchandises, dans la décision prise par la grande entreprise d'externaliser certaines fonctions auprès de PMI. Situés volontairement dans une démarche de type recherche-action, nous nous sommes appuyés pour cela sur de nombreuses enquêtes de terrain réalisées au sein de l'industrie agro-alimentaire française.

En effet, il est hors de propos ici de vouloir procéder à des généralisations hâtives, mais ce secteur a acquis, bien avant d'autres, une importante maturite logistique. Or, la PMI se charge très souvent de fabriquer «à sa façon» des produits finis pour le compte de prescripteurs. Ceux-ci sont alors conduits à les insérer efficacement dans leur système de distribution physique.

La convergence entre maturité logistique et stragégie de sous-traitance n'est pas, selon nous, fortuite. Elle témoigne de l'apparition de nouvelles formes de création de la valeur qui échappent aux modèles de l'intégration verticale ou de la croissance externe. Reste alors à ébaucher ce que pourraient être, non un modèle alternatif, mais les options stratégiques choisies (sous contrainte) par les firmes industrielles. Nous nous attacherons, par conséquent, d'une part à resituer les manoeuvres de la grande entreprise dans une dimension organisationnelle plus large et, d'autre part, à évaluer l'influence de ces manoeuvres sur la généralisation de la forme PME. 


\section{Vers un recentrage de la grande entreprise}

En rupture avec les discours prônant l'intégration verticale, des entreprises industrielles ont conscience de ne pas exceller dans toutes les tâches qui concourent à l'élaboration d'un bien dont pourtant elles assurent la commercialisation. Des activités périphériques comme l'entretien des équipements, la restauration du personnel et son ramassage par transport collectif, ... ont déjà donné lieu à une extériorisation auprès de sociétés spécialisées intervenant pour le compte d'un donneur d'ordres. Mais le phénomène s'accélère apparemment avec le déclenchement de la crise économique pour toucher des opérations en prise directe avec la «chaîne de valeurn.

L'heure est, semble-t-il, au recentrage de la grande entreprise qui internalise sélectivement certaines séquences de production ou de commercialisation en conservant le contrôle des séquences sous-traitées. Nous verrons que pour des industries comme l'agro-alimentaires, très sensibles aux consommations finales, la maîtrise de la distribution physique devient dès l'instant essentielle.

\subsection{La «chaîne de valeur», portée et limites}

Un des effets majeurs de la crise économique est sans hésitation la remise en question de nombreuses positions acquises sur des segments de marché. Plus particulièrement perceptible pour les biens de grande consommation, ce processus est entre autres caractérisé par l'affaiblissement de rentes de situation tandis que des savoir-faire de production se banalisent. La demande finale devient plus instable alors que des fabrications sont à la portée de PMI venant déstabiliser régionalement de puissants oligopoles.

Ce renouvellement du tissu industriel par l'arrivée de nouveaux entrants est très important pour la vitalité de l'économie, mais intervient dans une phase de stagnation-ou de légère croissance-de la consommation transformant laconcurrence inter-capitaliste en un jeu à somme nulle. Toute part de marché supplémentaire acquise par un compétiteur tend à réduire celle d'un ou de plusieurs autres. Pour les entreprises dominant un marché, le défi est donc double :

- Continuer à se démarquer des challengers par une politique marketing de différenciation;

- développer un avantage comparatif (notamment au niveau des coûts) vis-à-vis de PMI qui se placent sur les franges du marché sans supporter de lourdes charges d'exploitation.

Les hyperfirmes diversifiées en amont et en aval ont pu relever un tel défi jusqu'à une période récente en jouant sur des effets de dimension tout le long d'une 
filière : Tate \& Lyle ou Unilever en sont de parfaites illustrations. Cela est moins vrai maintenant. Ces hyperfirmes constatent peu à peu qu'il n'est plus possible de maîtriser correctement l'intégralité de ce que Porter (1985) nomme la «chaîne de valeur», c'est-à-dire l'ensemble des activités destinées à concevoir, fabriquer, commercialiser, distribuer et soutenir un produit.

Le renversement conceptuel est de taille. En effet, les modèles d'analyse industrielle ont, par le passé, décomposé des domaines d'actions stratégiques pour insister sur leur nécessaire liaison dans une même structure afin de récolter les fruits de multiples synergies et autres économies de champ. Or, Porter (1985), démontre que l'avantage concurrentiel ne repose plus mécaniquement sur ces politiques intégratives cumulant maillons sur et sous-efficients. Afin d'éviter «l'enlisement dans une voie médiane», il préconise trois stratégies :

- La domination par les coûts, lorsque cela s'avère praticable (économies d'échelle, accès préférentiel aux matières premières, ...);

- la différenciation (sur le produit, sur le système de distribution, sur l'approche marketing, ....);

- la concentration sur un segment cible.

Que dire de ces trois stratégies génériques? Outre leur simplisme, elles sont empreintes, comme l'ensemble du modèle de Porter (1985) d'ailleurs, d'un caractère normatif évident qui réduit leur portée. Mais surtout, elles présupposent qu'il suffit d'appliquer des solutions sommaires pour résoudre des problèmes complexes. Rien n'est moins sûr! En fait, la «chaîne de valeur» n'est qu'un instrument de décomposition analytique des activités participant, au sens large, à la création de valeur. De là à prescrire une des trois stratégies (et seulement) pour chaque maillon pertinent, il y a un pas qu'on ne peut franchir. Pour nous, c'est la limite majeure de l'approche éminemment empirique de Porter (1985).

En d'autres termes, la chaîne de valeur apparaît particulièrement stimulante en tant que description des activités principales et des activités de soutien de telle ou telle firme. Elle démontre que la situation concurrentielle n'est pas identique pour les divers maillons. Mais transposés sans vigilance, les emprunts au modèle de Porter (1985) risquent d'appauvrir la complexité des relations d'échange.

D'une part, l'auteurn'examine pas les stratégies d'alliance ou de coopération entre la grande entreprise et ses partenaires (Martinet, 1988). D'autre part, il écarte - ou sous-estime - la nécessaire cohérence du tout, c'est-à-dire l'articulation obligée entre les maillons, ceux qui restent internalisés et ceux qui sont sous-traités (spécialement auprès de PMI). Or, est-il possible de repenser le recentrage des firmes industrielles sans évoquer la gestion coordonnée de l'intégralité de la chaîne de valeur? 


\subsection{Sous-traltance et coûts de transaction}

Le processus de recentrage suppose, en définitive, que la grande entreprise «concentre ses efforts sur le maintien ou le développement de ce qu'elle considère comme ses compétences distinctives par rapport à la concurrence» (Tarondeau, 1982), quitte à externaliser les séquences qu'elle juge secondaires.

D'un point de vue théorique, elle est alors amenée à comparer systématiquement les avantages et désavantages respectifs de l'intégration verticale et des relations marchandes. À cette fin devront être évalués les coûts de transaction inhérents à l'échange. Ce dernier est un acte complexe, facteur d'incertitude, qui oblige à déterminer un contrat et donc implique à la fois de multiples négociations et des investissements spécifiques pour son exécution (Rainelli, 1989).

Si l'on suit ce raisonnement, l'achat de biens, plutôt que leur production, résulte d'un calcul économique qui intègre deux paramètres: les coûts de transaction et les savoir-faire possédés par des firmes extérieures. Lorsque les coûts diminuent vis-à-vis d'agents ayant la ou les compétences recherchées, par exemple grâce à une proximité urbaine (Cappellin, 1988), il devient plus facile d'externaliser plusieurs séquences. Inversement, on en conservera d'autres dont l'avantage comparatif en termes de coûts de transaction est défavorable.

Il ne faudrait pas en conclure imprudemment que la PMI est passive, voire sur-déterminée, dans sa relation avec le donneur d'ordres. Placée à l'inter-connexion de plusieurs chaînes de valeur, elle est au contraire capable de modifier le champ concurrentiel à son profit, par exemple en investissant plus aisément dans des équipements productiques (ce qui lui permettra de transformer son fond productif en fonction des besoins singuliers de ses donneurs d'ordres). Ainsi, autant la grande entreprise que la PMI pro-active peuvent tirer avantage de la relation fournisseur/ client, ... même si c'est à la grande entreprise qu'échoit le privilège d'organiser et de piloter les opérations sous-traitées.

En effet, l'externalisation requiert bien des capacités accrues de commande pour éviter tout blocage dans le fonctionnement de la chaîne de valeur: la «cohérence du tout», à laquelle nous avons fait allusion, s'appuie sur des rapports contractuels précisément normés entre organisations non hiérarchiques. Outre la réduction des coûts de transaction, il s'agit d'une condition nécessaire - mais non suffisante - pour donner un caractère opératoire aux concepts forgés par Porter (1985) et, globalement, comprendre pourquoi la PMI est maintenant un acteur essentiel des stratégies d'entreprises. 


\section{PMI et manoeuvres logistiques}

Forme nouvelle de la sous-traitance, à laquelle de nombreux observateurs dénient le statut, le façonnage est une illustration concrète des relations marchandes se nouant entre un donneur d'ordres et des PMI effectuant pour leur compte la fabrication d'une marchandise : l'intervenant extérieur prend en charge l'intégralité du procès de production aboutissant à un produit fini dont la grande entreprise peut achever la composition (par exemple, l'étiquetage).

Obéissant à une philosophie de la PMIsation, certaines firmes en arrivent alors à désintégrer leurs structures en unités autonomes de petite dimension. Le pilotage des flux entre ces éléments spatialement et juridiquement disjoints appelle une démarche de gestion nouvelle, la logistique.

\subsection{Le rolle du système de distribution physique : exemples}

La maîtrise de la circulation physique des marchandises est le support sur lequel reposent les pratiques d'extemalisation opérés dans le secteur agro-alimentaire. Plus précisément, seule une distribution physique performante autorise la «mise en normes» de la PMI à la logique de circulation désormais dominante. Deux exemples illustratifs :

\section{Cas $n^{\circ} 1$}

Buitoni France dispose de deux organisations productives, des usines en propre et des gammes sous-traitées. Environ un quart des fabrications sont réalisées par des petites entreprises nationales ou étrangères auxquelles la société propose la formule des produits, fournit l'ensemble des demi-produits (étiquettes, boîtes et emballages, ...) et procède à la commercialisation sous marque «Buitoni». Afin de mieux intégrer ces productions externalisées, la firme a réorganisé, en 1986, son système de distribution. Un entrepôt central a été construit près de la plus importante unité de production, à Orange, pour traiter l'intégralité des références. Il est relayé par des plates-formes gérées par des tiers, ayant une fonction expéditrice (livraisons finales) et surtout réceptrice/collectrice des marchandises provenant des différents sous-traitants industriels. Ces plates-formes constituent des «courroies de transmission» entre les productions géographiquement disséminées et le dépôt centralisateur : la diffusion nationale des petites gammes est assuré au moindre coût par ce système ambivalent réception/expédition qui génère une organisation industrielle se développant en réseau concentrique de PMI. Ainsi, le sous-traitant ne s'apparente qu'à un katelier» de la chaîne de valeur du donneur d'ordres. Celui-ci gère les tâches connexes comme la composition de lots promotionnels, l'étiquetage de «boîtes blanches», ... 


\section{Cas $n^{\circ} 2$}

La Société Française d'Alimentation Saine («La Vie Claire») vend des produits non dénaturés dont l'approvisionnement est en grande partie effectué par des fournisseurs sur la base d'un strict cahier des charges (et de «contrats de culture» pour les fruits et légumes). La société propose ainsi 900 références par familles de produits (jus de fruits, confitures, céréales, fruits secs, pâtes, ...) distribuées par un réseau de franchisés. La Direction Logistique joue un rôle fondamental puisqu'elle gère d'une part les achats et les unités de production intégrées, et d'autre part, la distribution physique. C'est donc elle qui vérifie la conformité des fabrications soustraitées aux critères de commercialisation, définis par ailleurs, la centralisation ayant lieu sur l'entrepôt de la région parisienne. L'imputation et la réalisation des transferts physiques sont cependant dévolues aux PMI gérant les livraisons d'approche franco entrepôt.

Environ $70 \%$ des marchandises étant stockées en moyenne une semaine, la Société Française d'Alimentation Saine impose à ses foumisseurs une régularité de réassortiment permettant de planifier les expéditions sur les franchisés. En effet, les points de livraison sont stables et peu nombreux, ce qui induit un délai de réaction assez bref en distribution finale. Toute la qualité de service repose sur la fluidité des transports d'approche et surl'efficience des manutentions intermédiaires.

Sans généraliser trop vite, on peut tirer de ces exemples un double constat. D'une part, de telles pratiques présentent certaines ressemblances avec lacoopération internationale inter-entreprises. Il s'agit sans doute de penser différemment le temps en économie de marché (Urban et Vendemini, 1988). Dans une période où le cycle de vie des produits a tendance à fortement se contracter, la grande entreprise élargit sa gamme et la diversifie en fonction de l'évolution volatile de la demande finale. Par la sous-traitance, le donneur d'ordres stabilise sur un horizon temporel plus long des couples produits/marchés tout en réduisant les risques liés à l'immobilisation des investissements matériels et immatériels. D'autre part, l'agro-alimentaire semble bien mû par une logique de flux, c'est-à-dire d'optimisation de la fluidité de la circulation physique. Ceci n'est pas en soi remarquable : la faible valeur ajoutée dégagée pousse à une rationalisation des opérations afin d'éviter toute inertie coûteuse, comme les sur-stocks de produits finis.

Les grandes entreprises creent finalement les conditions optimales d'insertion des PMI dans leur système de circulation pour accroître leur flexibilité commerciale. Allantencore plus loin, certaines d'entre elles en viennentà généraliser la forme PME en décomposant la chaîne de valeur auparavant intégrée en cellules juridiquement indépendantes. Ce mouvement, que nous avons dénommé par analogie «scissiparité» (Paché et Paraponaris, 1987), permet selon ses promoteurs de lutter contre le gigantisme des unités et de mieux contrôler les coûts par la génération de multiples centres de profit. Pour tout dire, les déséconomies de gestion conduisent les firmes 
géantes à repenser leur développement hors de l'ancienne logique conglomérale (Julien et Marchesnay, 1988). C'est le cas de Galac Claudel qui a divisé en 1984 sa structure de production : seule l'usine de Lons-le-Saunier, fabriquant le fromage fondu «Grosjean», appartient toujours au groupe tandis que six autres unités sont aujourd'hui autonomes.

La question est alors de savoir s'il existe une filiation, au niveau des systèmes de circulation mobilisés, entre le recours à la PMI façonnière et la création de PMI par un processus de «fragmentation».

\subsection{Apport de la logistique à l'analyse industrielle}

Décrivant la systématisation de la forme PME, qu'il appelle PMIsation, De Banville (1984) évoque «le réarrangement continu qu'opère le capitalisme dans son adaptation aux conditions socio-économiques de sa reproduction». Cette approche est sans doute fructueuse mais elle réclame une nette distinction entre le mouvement de captation, tel qu'il est décrit par les deux cas présentés au-dessus, et le mouvement de «répulsion» ou scissiparité. En effet, les PMI nées du deuxième mouvement ontelles véritablement le statut afférent, hormis la petite dimension? Ou ne bénéficientelles pas des savoir-faire déjà accumulés par la grande entreprise ?

Ces interrogations sont importantes, particulièrement vis-à-vis des transferts physiques (cf. ci-dessous), mais elles renvoient également au rôle de la PMIsation dans la stratégie d'entreprise. Il semblerait que l'extériorisation est un moyen de réaliser l'audit d'une organisation et mesurer par là l'efficacité relative de chacun des maillons. Afin qu'aucun d'entre eux ne puisse bénéficier par rétention de gains de productivité issus d'un autre point de la chaîne, il y aurait en conséquence segmentation par centres de profit, d'où la possibilité d'évaluer finement la valeur ajoutée générée.

Nous n'entrerons pas plus avant dans l'analyse en retenant ce qui, à notre niveau d'investigation, est l'enseignement majeur de la PMIsation. La grande entreprise fait sous-traiter à des PMI des séquences de production selon des normes et des formats industriels qu'elle a rigoureusement définis, et que la PMI pourra progressivement modifier par des innovations technologiques récurrentes. La «recomposition» (le fonctionnement cohérent) de la chaîne de valeur ne se réalisera que parl'intermédiaire d'un management logistique régulant les flux entre les unités. Son rôle sera de déclencher juste à temps les différentes opérations, par exemple le lancement d'une série, mais aussi de formuler le réseau physique dans lequel se placent ces unités (Colin et Paché, 1988).

En ce sens, il nous parait vain de conceptualiser la PMIsation sans se référer au préalable à la démarche organisationnelle qui la soutient et permet de soutenir, voire diminuer, les coûts de transaction. Sous cette condition, il devient possible de voir que le recours à la forme PME par la grande entreprise obéit, dans l'industrie agro-alimentaire, à un modèle dual. 
a) Le processus de captation repose sur des systèmes relais de type entrepôt (Buitoni). On a de fait, un circuit d'approvisionnement à deux niveaux : les flux physiques sont transmis jusqu'au(x)point(s) de pénétration du système logistique, puis sont ensuite éclatés en fonction des besoins commerciaux. Ces points d'entrée spécialisés par gammes de produits, par familles logistiques, ... peuvent être gérés en propre ou par des prestataires extérieurs (Paché, 1989).

Cette organisation n'est pleinement opérationnelle que si l'articulation des maillons de la chaîne de valeur réduit - ou supprime - les arrêts de produits finis du lieu d'expédition jusqu'au point de vente. Il y aura à la limite autant de points d'entrée que de références commerciales si la contrainte majeure de qualité de service rendu à la clientèle est scrupuleusement respectée. On sait notamment qu'une absence de ponctualité dans les livraisons peut conduire en France à un déréférencement par les distributeurs commerciaux...

b) L'opposition paraît franche avec la philosophie de la scissiparité. En effet, la conception des systèmes de circulation (tournées de livraison informatisées, choix des transporteurs, utilisation conjointe de plates-formes, ...) est alors pensée par le «noyau» central qui se prémunit des risques liés à l'éclatement juridique. Ce constat concorde au demeurant avec les pratiques des G.I.E. de distribution (Générale Biscuit, Sogasi-Bongrain, Moët-Hennessy, ...) qui centralisent volontairement les flux de produits finis provenant des différentes filiales vers des lieux de regroupement/éclatement très souvent reliés directement à une voie ferrée ou équipés pour gérer des techniques de transport combiné (conteneurs terrestres). Ainsi, Galac Claudel, ayant mis en oeuvre une politique de scissiparité (cf. ci-dessus), conserve le réseau de distribution physique érigé des 1975.

Sur le plan logistique donc, il n'y a pas véritablement rupture dans la mesure où la fragmentation maintient les liens juridiques (mais les distend) entre le «noyau» et les satellites qui gravitent autour de lui. Tout dérapage au niveau des coûts aura, il est vrai, un impact immédiat sur la compétitivité globale de la chaîne de valeur. De plus, en période de turbulence, si le besoin d'externaliser ce qui était internalisé devient plus grande, il s'accompagne également d'un besoin accru de coordination des opérations (Wright, 1986).

Rien ne permet d'avancer malgré tout que la scissiparité soit totalement exclusive de relations purement marchandes. Les entretiens menés auprès de responsables logistiques placés dans cette situation montrent que la grande entreprise envisage très souvent l'autonomisation totale de ses filiales/«PMI» lorsqu'elles seront capables de formaliser leurs exigences en matière de transferts physiques. Ce qui passe par un apprentissage logistique diffus et non plus exclusivement focalisé dans les directions fonctionnelles. 


\section{Conclusion}

Nous nous étions proposés de réfléchir sur des modes d'insertion de la PMI dans le management logistique de la grande entreprise. Cette réflexion nous a permis de formuler les logiques d'action en vigueur dans l'industrie agro-alimentaire française où la distribution physique joue un rôle central dans les manoeuvres stratégiques actuelles. Onassiste ainsi à la sous-traitance de sous-ensembles complets de produits auprès de PMI ayant pour mission originelle d'industrialiseret d'assembler des marchandises conçues et distribuées par d'autres.

Il n'est certes pas question d'extrapoler des résultats ponctuels à toutes les branches d'activité. Néanmoins, la lecture de revues professionnelles et l'étude de cas issus du textile, de la micro-électronique, ... témoignent d'étonnantesconvergences d'analyse. Et certains auteurs n'hésitent plus à faire allusion aux «firmes solaires» qui prennent le contre-pied des anciennes logiques d'intégration (Piore et Sabel, 1984), ce qui rejoindrait plutôt notre propos.

En fait, il nous semble que le terrain est suffisamment neuf et original pour susciter un ensemble de travaux dans le champ de la gestion et de l'analyse industrielle. À ce titre, deux voies seraient à explorer :

- La mesure de la corrélation entre processus de désintégration verticale et émergence d'une logistique performante, etl'explication d'éventuelles distorsions inter-sectorielles;

- l'impact du recentrage de certaines grandes entreprises sur la qualification technologique et managériale des PMI en situation de sous-traitance.

\section{BIBLIOGRAPHIE}

Cappelin, R., (1988), «Transaction costs and urban agglomeration», Revue d'Economie Régionale et Urbaine, $\mathrm{n}^{\circ} 2$, p. 261 à 278.

Colin, J. et Paché, G., (1988), La logistique de distribution, Chotard et Associés Éditeurs, Paris.

De Banville, E., (1984), L'entreprise entre la PMIsation et l'évolution des réseaux de solidarité, CRESAL, Saint-Étienne, Juillet.

Julien, P.A. et Marchesnay, M., (1988), La petite entreprise : principes d'économie et de gestion, Vuibert Gestion, Paris.

Marchesnay, M., Fauré, R. et Mathié, B., (1979), «L'ombre des grands», Revue Française de Gestion, $\mathrm{n}^{\circ} 22$, p. 108 à 115.

Martinet, A.C., (1988), «Les discours sur la stratégie d'entreprise», Revue Française de Gestion, n $^{\circ} 67$, p. 49 à 60.

Paché, G., (1989), «Conflits et pouvoirs dans la commercialisation des produits agroalimentaires : industriels, distributeurs, prestataires logistiques», Economies et Sociétés, Série Développement agro-alimentaire, Tome XXIII, nீ $^{\circ}$ (à paraître). 
Paché, G. et Paraponaris, C., (1987), «Réorganisation du capital industriel formes liées de la gestion de production», Economies et Sociétés, Série Sciences de Gestion, Tome XXI, $\mathrm{n}^{\circ}$ 6, p. 151 à 177.

Piore, M.J.et Sabel, C.F., (1984), The Second Industrial Divide, Basic Books Inc., New-York.

Porter, M., (1985), Competitive Advantage, The Free Press, New-York.

Rainvelli, M., (1989), Economie industrielle, Dalloz, Paris.

Tarondeau, J.C., (1982) Produits et technologies, Dalloz Gestion, Paris.

Urban, S. et Vendemini, S., (1988), Les entreprises industrielles italiennes et la coopération internationale, CESAG (Université Robert Schuman), Strasbourg, Octobre.

Wright, M., (1986), «The make-buy decision and managing markets : the case of management buy-outs», Journal of Management Studies, Vol. XXIII, n 4, p. 443 à 464. 